\title{
Intravenous lidocaine and postoperative bowel motility in laparotomies for generalized acute peritonitis: double-blind randomized clinical trial at souro-sanou teaching hospital in Bobo-Dioulasso, Burkina Faso
}

\begin{abstract}
Introduction: The reduction of the duration of postoperative paralytic ileus remains a major challenge for anesthetists in digestive surgery. Different strategies have been proposed including the use of intravenous lidocaine perioperatively. The objective of this study was to evaluate the effect of intravenous lidocaine on postoperative bowel motility in urgent abdominal surgery.

Method: This was a randomized, double-blind, 6-month clinical trial at Souro-Sanou teaching hospital in Bobo Dioulasso, Burkina Faso. The patients included in the study were separated after randomization into 2 groups, one receiving lidocaine intravenously perioperatively and the other receiving saline (placebo). The main evaluation criteria were the intestinal transit recovery time and the length of the hospital stay. Secondary endpoints included pain control, opioid need, and mortality.

Results: Out of 194 patients who had laparotomy for generalized acute peritonitis during the study period, 65 patients were analyzed, including 31 in the placebo group and 34 in the lidocaine group. The average age in patients was 39.4 years. The average time taken to treat patients in our study was $17 \mathrm{~h} \pm 2.7$; with extremes of $1 \mathrm{~h}$ and 144 h. The average consumption of sufentanil was $20.6 \mu \mathrm{g}$ and $24.1 \mu \mathrm{g}$ respectively in the lidocaine group and the placebo group, a reduction of $14.5 \%$ in the intraoperative consumption of opioids $(\mathrm{p}=0.03)$. At the 48 th postoperative $\mathrm{h}$, no patients in the placebo group had stooled against two patients in the lidocaine group $(\mathrm{p}=0.07)$. The average hospital stay was 6.4 days $(\mathrm{p}=0.8)$ in both groups. The overall mortality was $10.8 \%$.

Conclusion: Perioperative intravenous lidocaine does not reduce the duration of postoperative paralytic ileus, but has a significant analgesic effect thus allowing significant morphine savings.
\end{abstract}

Volume 10 Issue 2 - 2018

\author{
Traore IA,' Barro SD,' Ki KB, ${ }^{2}$ Zongo G, ${ }^{3}$ \\ Guibla I' \\ 'Souro Sanou teaching hospital, Burkina Faso \\ ${ }^{2}$ Charles De Gaulle teaching hospital, Burkina Faso \\ ${ }^{3}$ Ouahigouya teaching hospital, Burkina Faso
}

Correspondence: Dr. Traoré Ibrahim Alain, assistant professor, anesthesiology and intensive care, Souro Sanou teaching hospital, POB: 314, Bobo-Dioulasso, Burkina Faso, Tel 002267|457580, Email itraore80@gmail.com

Received: February 28, 2018 | Published: March 21, 2018

Keywords: Intravenous, lidocaine-postoperative, ileus-generalized, acute peritonitis

\section{Introduction}

Reducing the duration of postoperative paralytic ileus remains a major challenge for anesthetists in digestive surgery. Numerous studies carried out as part of an accelerated postoperative rehabilitation program $^{1,2}$ have shown the beneficial effect of intravenous lidocaine perioperatively, due to the discovery in recent years of its multiple effects, in particular the reduction of peroperative opioids consumption and the duration of postoperative paralytic ileus ${ }^{3-6}$ In sub-Saharan Africa, particularly in Burkina Faso, acute generalized peritonitis is one of the most common surgical emergencies. In fact, in 2015 , it accounted for $25 \%$ of surgical emergency room admissions at Souro-Sanou teaching hospital in Bobo Dioulasso. ${ }^{7}$ Worse, it was the emergency that required the longest hospital stay in 2014 at the Yalgado Ouedraogo Hospital of Ouagadougou in Burkina Faso. ${ }^{8}$ The objective of this study was to evaluate the effect of intravenous lidocaine on postoperative bowel motility to help improve the prognosis of patients with laparotomy for acute generalized peritonitis.

\section{Methods}

After approval of the National Ethics Committee, this study was conducted over a 6-month period from May to November 2016 at Souro-Sanou teaching hospital in Bobo-Dioulasso, Burkina-Faso. Adult patients at least 18 years of age and up to 70 years of age, classified as ASA 2 or 3 after pre-anesthetic evaluation, received laparotomy for acute generalized peritonitis after giving their consent were included in the study. We excluded from our study, patients classified ASA 4 or 5 , those in septic shock, those with a clearance of creatinine less than $50 \mathrm{ml} /$ min according to the Cockcroft-Gault formula, as well as pregnant women. The same applies to patients who have been misdiagnosed, those who have not been followed by the protocol, patients who are already suffering from hepatocellular insufficiency or who have known allergy to lidocaine, and finally those who are in an impossibility of giving a free and enlightened agreement. The objective of our study was to evaluate the effect of intravenous lidocaine perioperatively on the recovery time of 
postoperative paralytic ileus. Thus, after inclusion in the study, we proceeded after adequate preoperative resuscitation to the assignment of patients by random draw with randomization envelopes sealed in 2 treatment groups. One group (lidocaine group) receiving $1.5 \mathrm{mg}$ bolus lidocaine after conventional fast-acting anesthetic induction, then 2 $\mathrm{mg} / \mathrm{kg} / \mathrm{h}$ intraoperatively and $1.3 \mathrm{mg} / \mathrm{kg} / \mathrm{h}$ during the first 24 hours post-operatively. The other group (placebo group) control receiving an equivalent volume of saline. Lidocaine and saline cannot be distinguished visually and presented in identical syringes. Other per and postoperative resuscitation measures were identical in both groups. A fact sheet previously prepared and tested from patients clinical records, anesthetic and operative protocols, as well as hospitalization records from general purpose resuscitation, general surgery and digestive services enabled us to collect data from our study. This data was then entered on the Epi Data version 3.1 software, cleaned and analyzed using the Stata software version 12 . The average values of the emission delay of the first gases and stools were compared by the student test. Postoperative pain assessed by rating the visual analog scale (EVA) at rest and cough, the consumption of halothane, total consumption of morphine in per and postoperative, the reinjection of opioids, the average length of hospital stay was compared by performing a 2-factor ANOVA. The frequency of occurrence of postoperative complications was compared using the chi-square test or the fisher test if expected values were less than 5 . The degree of significance was set for a p-value less than 0.05 .

\section{Results}

The monocentric nature of this study limits its external validity. But the double-blind randomization of patients operated for acute generalized peritonitis made it possible to obtain results statistically closer to reality in our context. Thus, out of 194 patients who had undergone a laparotomy for acute generalized peritonitis during the study period, 102 patients were included in our study. At randomization, 65 patients were analyzed, including 31 in the placebo group and 34 in the lidocaine group. At the socio-demographic level, patients in our study population were relatively young with a mean age of 39.4 years with extremes of 18 and 69 years. The most represented age group was 25-49 in both groups. The sex ratio was 2.8 in our population in favor of men (48 men for 17 women). In our study population, out-of-school participants were in the majority with $64.3 \%$ in the lidocaine group and $70.6 \%$ in the placebo group. The mean time to management in our study was $17 \mathrm{~h} \pm 2.7$; with extremes of $1 \mathrm{~h}$ and $144 \mathrm{~h}$. Less than a third of our patients were treated within 6 hours of admission. On clinically, the patients in our study were mostly classified as ASA $2(73.8 \%)$. Gastric ulcer perforation was the etiology of the most represented acute generalized peritonitis $(52.9 \%)$ in our study sample. Therapeutically, the average duration of interventions was 70.8 minutes \pm 26 with extremes of 30 and 150 minutes. In our series, the average consumption of sufentanil was 20.6 $\mathrm{mcg}$ and $24.1 \mathrm{mcg}$ respectively in the lidocaine group and the placebo group, a reduction of $14.5 \%$ in the intraoperative consumption of opioids ( $\mathrm{p}=0.03$ ). Used on average in 2 groups to $1 \%$ to the incision and during the first 30 minutes of the procedure, the concentration of halothane in the $60^{\text {th }}$ minute was $0.65 \%$ in the lidocaine group and $0.68 \%$ in the placebo group. As for postoperative pain, the visual analog scale (EVA) performed successively at the 6th, 12th and 24th hour found a greater decrease in pain in the lidocaine group $(\mathrm{p}=0.02)$. Figures 1 and 2 show the resting and coughing pain scores respectively for lidocaine and placebo patients.

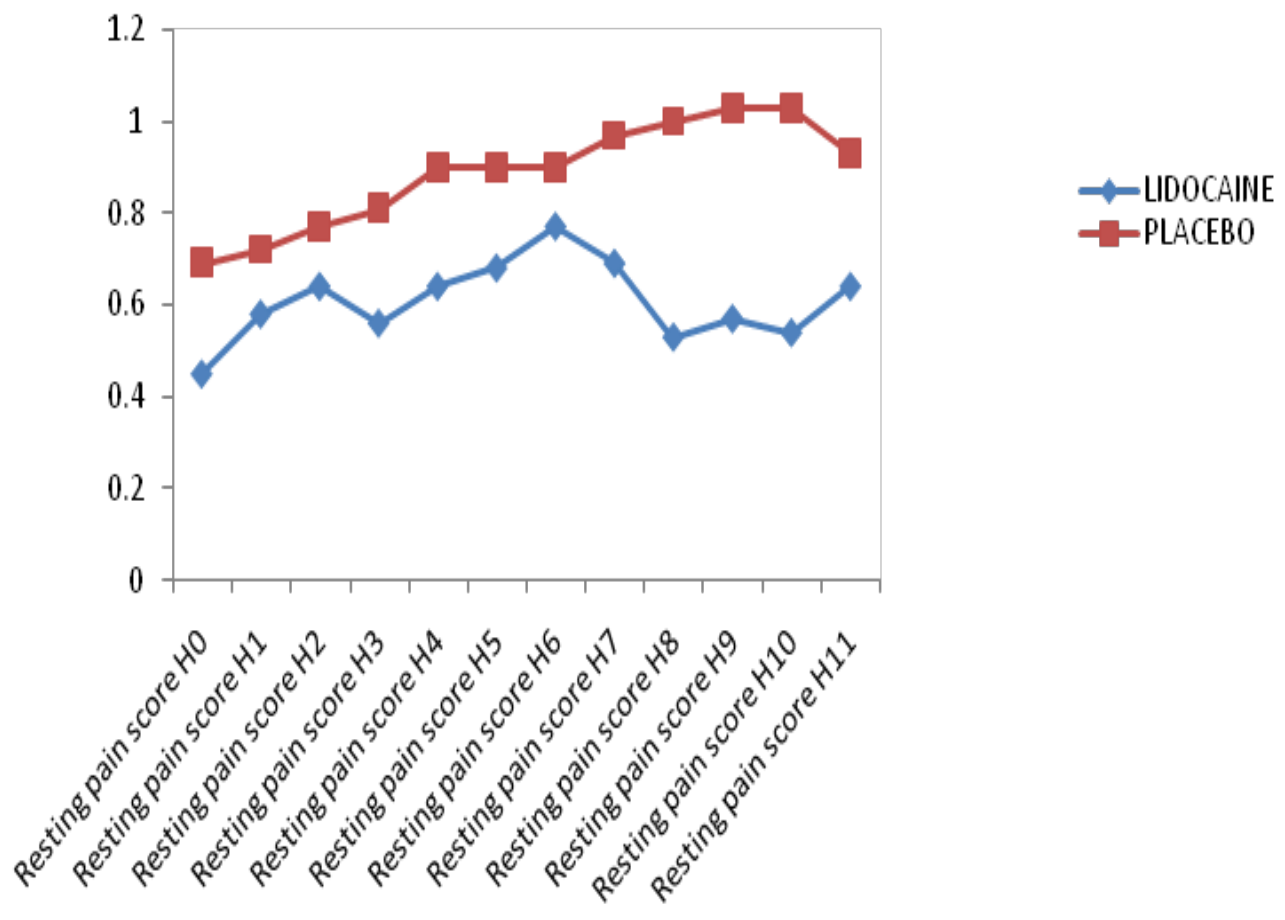

Figure I Resting pain scores.

Citation: Traore IA, Barro SD, Ki KB, et al. Intravenous lidocaine and postoperative bowel motility in laparotomies for generalized acute peritonitis: doubleblind randomized clinical trial at souro-sanou teaching hospital in Bobo-Dioulasso, Burkina Faso. Anesth Crit Care Open Access. 2018;10(2):67-7I. DOI: $10.15406 /$ jaccoa.2018.10.0036| 


\section{Means of caugh pain score}

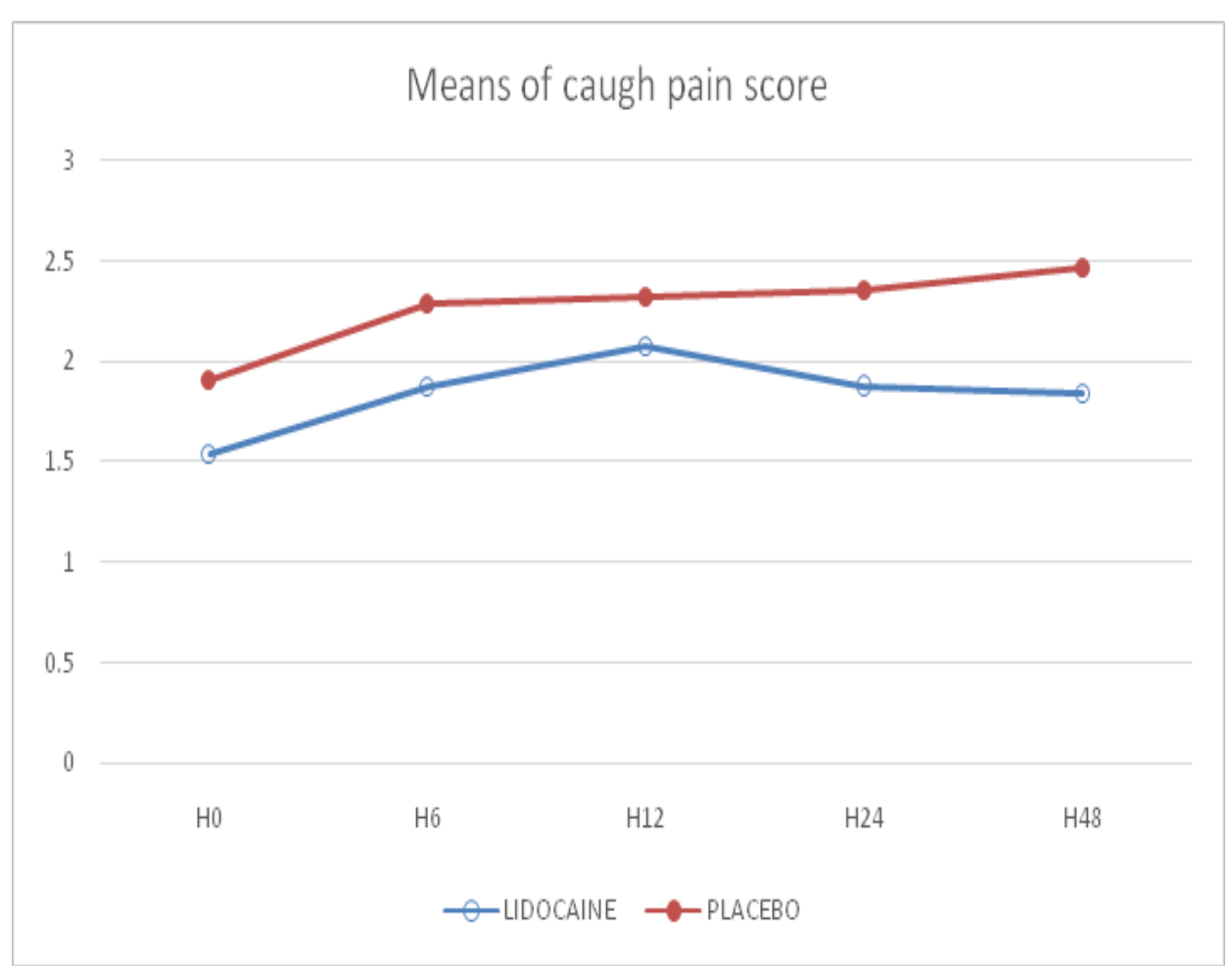

1.5

Figure 2 Cough pain scores.

At the 48th postoperative time, no patients in the placebo group had stooled against two (2) patients in the lidocaine group $(\mathrm{p}=0.07)$. The frequencies of the various transit restart parameters are presented in Table 1.

Table I Frequencies of the various transit restart parameters at the 48th hour

\begin{tabular}{llllll}
\hline & Groups & $\begin{array}{l}\text { Hydroaeric } \\
\text { noises }\end{array}$ & First gases & $\begin{array}{l}\text { First } \\
\text { stool }\end{array}$ & P \\
\hline $\begin{array}{l}\text { At the } \\
\mathbf{4 8}^{\text {th }}\end{array}$ & Lidocaine & $15.4 \%$ & $12.0 \%$ & $10.5 \%$ & \\
hour & Placebo & $6.1 \%$ & $6.1 \%$ & $0.0 \%$ & $\mathrm{P}=0.07$ \\
\hline
\end{tabular}

Of the 65 patients included in our study, seven died including $85.7 \%$ in a septic shock; an overall mortality of $10.8 \%$. The average length of hospital stays presented in the following Table 2 was identical in the 2 groups ( 6.4 days) but not significant $(p=0.8)$ with extremes of 3 and 12days (lidocaine), and 3 and 26 days (placebo).

Table 2 Rehabilitation and length of stay

\begin{tabular}{llll}
\hline & lidocaine & Placebo & $\mathbf{p}$ \\
\hline GET UP (hours) & $\mathbf{9 9 . 9}$ & 89.7 & 0.4 \\
POWER (hours) & $\mathbf{1 0 5 . 6}$ & 93 & 0.4 \\
STAY (days) & $\mathbf{6 . 4}$ & 6.4 & 0.8 \\
\hline
\end{tabular}

\section{Discussion}

The average age of patients in our sample was 39.4 years old. Studies in Burkina Faso and in the sub region reported a lower average age. ${ }^{7,9}$ These studies, unlike the present, included all patients without age criteria, but are unanimous in recognizing that acute generalized peritonitis is a pathology of young adults in our context. We found a male predominance in agreement with the data of the literature. ${ }^{9-13}$ In Burkina Faso, despite the high proportion of women $(52 \%)$ in the general population, women retain low decision-making power in the couple due to sociocultural constraints. ${ }^{14}$ Indeed, the Demographic Health Survey (DHS) 2015 in Burkina Faso reveals that in $79 \%$ of cases, the decision on women's health care is taken by the joint. ${ }^{14}$ Thus, in case of illness, the woman must refer to the decision of her spouse before going to a health center which could explain the male predominance found in our study. For some authors, the reasons for this male predominance remain uncertain and suggest instead that the lack of systematic care of women for abdominal and / or pelvic pain is linked to the perception that they perceive them as "normal". ${ }^{7}$ In our series, most patients received late treatment with an average delay of $17 \mathrm{~h} \pm 2.7$, thus corroborating those of other African series. ${ }^{9} 15$ However, acute generalized peritonitis is a serious surgical emergency whose prognosis is highly dependent on the precocity of care and should therefore benefit from adequate care within 6 hours of onset of the disease. The sub-Saharan countries are distinguished at the health level by late resorting to the care of modern medicine. Also, the difficulty of some patients to honor prescriptions, the small number of teams of anesthetists and surgeons would justify this long period of care. Although most patients in our series were classified as ASA 2, perforation of gastric ulcer was the most recovered etiology, unlike the Kassene series in Togo, Harouna in Niger, Da and Ouangré in Burkina, which found etiology post-typhus ileal perforation respectively in $64.2 \% ; 39 \% ; 31.2 \%$; and $42.5 \% .{ }^{9}, 15,7,16$ This difference could be explained by the existence of a period of Muslim fasting during our study period. In addition, post-typhoid ileal peritonitis is correspondingly more complicated with septic shock, which is a noninclusion criterion in our series..$^{9,17}$

Lidocaine iv and morphine sparing. Our study found a significant savings of opioids in patients who received intravenous 
lidocaine $(\mathrm{p}=0.03)$. The same is true for Kaba, ${ }^{1}$ who found that the use of intravenous lidocaine perioperatively would reduce opioid consumption by $50 \%$ in the 24 hours postoperative laparoscopic colectomy in programmed surgery. For McCarthy, this reduction would be on average $85 \%$, whatever the type of abdominal surgery. ${ }^{2}$ But the urgency of the intervention and the surgical approach recognized as very invasive and a source of greater postoperative pain in our series could explain the low opioid gain in comparison with the Kaba team. In contrast to these three teams, other series found in the literature, notably that of Sanders in 2014 in a randomized double-blind study, showed that there was no significant difference in terms of opiods consumption between the patients who benefited perioperative continuous administration of intravenous lidocaine and those of the placebo group during laparoscopic colectomies. ${ }^{18-21}$ However, unlike Sanders who maintained narcosis with propofol, we used halothane in our series when we know that halogenated have a proper anti-nociceptive effect via a central action by blocking NMDA receptors. This could result in synergistic action and a significant benefit in terms of analgesia. This could explain the significant results obtained in our study concerning opioids saving despite a less clear difference in terms of quantity.

Lidocaine IV and postoperative pain: In our series, the postoperative pain assessed by the visual analog scale (EVA) at rest and cough found a significantly higher score in the placebo group $(p<0,05)$. Other authors have reached the same results. It is essentially that of Kopper but especially of Vigneault in the Canadian Journal of Anesthesia which in a meta-analysis of 29 studies and 1,754 patients also found a weighted mean difference of the EVA in favor of the patients under intravenous lidocaine with a morphine gain of 8.44 $\mathrm{mg} .{ }^{22,23}$ For the Kaba team, this morphine gain could go up to $14 \mathrm{mg}$ $(\mathrm{p}=0.005) .{ }^{1}$ The presence of visceral pain and severe inflammation may be related to a greater interest in lidocaine. ${ }^{21,24}$ Acute generalized peritonitis meets these criteria, hence the significant effect of intravenous lidocaine on analgesia in our study. ${ }^{21,25}$

Lidocaine IV and resumption of digestive transit: The data collected in our series in the first 48 hours postoperative showed a beneficial but not significant effect of lidocaine on the resumption of digestive transit $(\mathrm{p}=0.07)$. This observation is similar to that of Harvey in 2009 who also found in a pilot study on intravenous lidocaine a recovery of the earlier but not significant postoperative intestinal motility $(\mathrm{p}=0.28)$ in the interventional group and with a recovery time postoperative shorter than in our series. ${ }^{26}$ In contrast, Kaba in 2007, in a study of laparoscopic colectomies found a very significant beneficial effect of intravenous lidocaine for the emission of the first gases (17 h $[11-24 h]$ vs. $28 \mathrm{~h}[25-33 \mathrm{~h}] ; \mathrm{P}<0.001)$, first stool $(28 \mathrm{~h}[24-37 \mathrm{~h}]$ vs. $51 \mathrm{~h}[41-70 \mathrm{~h}], \mathrm{P}=0.001)$, and therefore a shorter recovery time $(\mathrm{p}=0.001) .{ }^{1}$ These two studies, unlike our series, were all performed in programmed surgery with its corollary of adequate preoperative preparation and especially in the framework of an accelerated postoperative rehabilitation program. Thus, the prior existence of a preoperative ileus related to pathology (acute generalized peritonitis), the permanent use of weak opiates for postoperative analgesia and especially the absence of an accelerated postoperative rehabilitation program operational at Souro-Sanou teaching hospital could constitute factors limiting the earlier recovery of transit found in our series.

Lidocaine IV and hospital stay: The duration of postoperative hospitalization was identical but not significant $(p=0.8)$ in the placebo and lidocaine groups in our series. In contrast, Kaba in his laparoscopic colectomy study and Harvey found a gain of about 1 day in favor of the lidocaine group, with $\mathrm{p}$ significance of 0.001 and 0.02 , respectively [1.26]. Sun in China, in a meta-analysis involving 21 randomized clinical trials concluded that perioperative intravenous lidocaine administration decreased by 0.71 days on average $(95 \%$ CI: $-1.35,-0.07) ; \mathrm{I}^{2}=37.3 \%$ ) the duration of hospitalization in the interventional group. ${ }^{24}$ The context of the emergency and especially the absence of operational postoperative rehabilitation program found in our series as well as in most African series ${ }^{9,15}$ could explain this difference.

\section{Conclusion}

Our study could not objectify a significant difference in the resumption of postoperative transit and thus a reduction in the length of hospital stay after perioperative administration of intravenous lidocaine. Nevertheless, it has been shown that the perioperative administration of intravenous lidocaine was accompanied by a significant analgesic effect and therefore significant morphine savings in patients after laparotomy for acute generalized peritonitis. Thus, to improve the prognosis of laparotomies for acute generalized peritonitis by reducing the duration of postoperative ileus, the intravenous lidocaine protocol could be introduced as part of an accelerated postoperative rehabilitation program.

\section{Acknowledgment}

None.

\section{Conflict of interest}

Authors don't have any conflict of interest.

\section{References}

1. Kaba A, Laurent SR, Detroz BJ, et al. Intravenous lidocaine infusion facilitates acute rehabilitation after laparoscopic colectomy. Anesthesiology. 2007;106(1):11-8.

2. McCarthy GC, Megalla SA, Habib AS. Impact of intravenous lidocaine infusion on postoperative analgesia and recovery from surgery: a systematic review of randomized controlled trials. Drugs. 2010;70(9):1149-1163

3. Herroeder S, Pecher S, Schönherr ME, et al. Systemic lidocaine shortens length of hospital after colorectal surgery: double-blinded, randomized, placebo-controlled trial. Ann Surg. 2007;246(2):192-200.

4. Lauwick S, KA, Joris J. Intravenous injection of local anesthetics, lidocaine: interest, limitations, use in practice. Web anesthesia. 2009;2009-3.

5. Attal N, Gaude V, Brewer L, et al. Intravenous lidocaine in central bread: a double-blind, placebo-controlled, psychophysical study. Neurology. 2000;54(3):564-74

6. Finnerup NB, Biering-Sorensen F, Johannes in IL, et al. (2005) Intravenous lidocaine relieves spinal cord injury pain: a randomized controlled trial. Anesthesiology 102:1023-1030.

7. Da D. Cyriac Medical thesis: Generalized acute peritonitis: epidemiological, clinical and therapeutic aspects at CHNSS BoboDioulasso about 369 cases. 2016;21:43.

8. Bonkoungou PZ, Zida M, Traore SS, et al. Postoperative ileus in the department of visceral and digestive surgery of the University Hospital Center Yalgado Ouedraogo of Ouagadougou. 
9. Kassegne I, Kanassoua KK, Sewa EV, et al. Management of acute generalized peritonitis at Kara University Hospital. Saranf. 18(2):115-21.

10. Sanou MJ. Surgical abdomens with CHNYO. Review of five years of activities of a general surgery department. Thesis Med. Ouagadougou, 1991.

11. Ouattara T, Bonkoungou B, Kambou T, et al. Severity factors of peritonitis at CHNSS Bobo-Dioulasso. About 47 cases. Annals of the University of Ouagadougou, 1994, series B, 44-49.

12. Nali MN, Bedaya-Ngaro S, Mandaba JL, et al. Typhic peritonitis: Studies of 43 cases observed at the National University Hospital Center of Bangui, from 1982 to 1986.Med. of Black Afrq, 1989;36(1):38-40.

13. Kafando RJ. Typhic perforations: clinical and therapeutic aspects. About 239 cases collected at CHNYO. Thesis Med. Ouagadougou, 1997;6.

14. National Institute of Statistics and Demography. Demographic Health Survey (DHS) Burkina Faso 2015.

15. Harouna YD, Abdou I, Saidou B, et al. Peritonitis in tropical environment, etiological particularities and current prognostic factors - About 160 cases Medicine of Black Africa 2001;48(3).

16. Ouangre E, Zida M, Bonkoungou PG, et al. Generalized Acute Peritonitis in Rural Areas in Burkina Faso, About 221 Cases, Rev. CAMES SANTE Vol.1, $\mathrm{N}^{\circ}$ 2, December 2013.

17. Kuo CP, Jao SW, KM Chen, et al. Comparison of the effects of thoracic epidural analgesia and iv infusion with lidocaine on cytokine response, postoperative pain and bowel function in patients undergoing colonic surgery. Br J Anaesth. 2006;97(5):640-6.

18. Herroeder, Pecher S, Schonherr ME, et al. Systemic Lidocaine Shortens Length of Hospital After Colorectal Surgery: A double-blinded, randomized, placebo-controlled trial. Ann Surg. 2007;246(2):192-200.
19. Lauwick S, Kim DJ, Mistraletti G, et al. Functional walking capacity as an outcome measure of laparoscopic prostatectomy: the effect of lidocaine infusion. Br J Anaesth. 2009;103(2):213-19.

20. Wu CT, Borel CO, MS Lee, et al. The interaction effect of perioperative co-treatment with dextromethorphan and intravenous lidocaine on pain relief and recovery of bowel function after laparoscopic cholecystectomy. Anesth Analg. 2005;100(2):448-53.

21. Saadawy IM, Kaki AM, Abd El Latif AA, et al. Lidocaine vs. magnesium: effect on analgesia after laparoscopic cholecystectomy. Acta Anaesthesiologica Scandinavica. 2010;54(5):549-56.

22. Koppert W, Weigand M, Neumann F, et al. Perioperative intravenous lidocaine has a positive effect on postoperative pain and morphine consumption after major abdominal surgery. Anesth Analg. 2004;98(4):1050-5.

23. Vigneault L, Turgeon A F, Side D, et al. Perioperative lidocaine intravenous infusion for postoperative pain control: a meta-analysis of randomized controlled trials. Can J Anaesth. 2011;58(1):22-37.

24. Sun Y, Li T, Wang N, Yun Y, et al. Perioperative systemic lidocaine for postoperative analgesia and recovery after abdominal surgery: a meta-analysis of randomized controlled trials. Dis Colon Rectum. 2012;(55):1183-94.

25. Naito Y, Tamai S, Shingu K, et al. Responses of plasma adrenocorticotropic hormone, cortisol, and cytokines during and after upper abdominal surgery. Anesthesiology. 1992;77(3):426-31.

26. KP Harvey, JD Adair, Isho M Robinson R. Can intravenous lidocaine decrease postsurgical ileus and hospital shorten stay in elective bowel surgery? A pilot study and literature review. Am JSurg. 2009;198(2):231-6. 